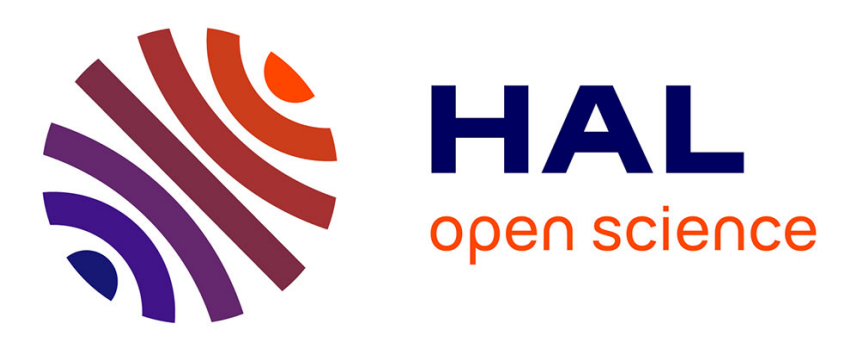

\title{
Indissociables et irréductibles : les rapports sociaux de genre, ethniques et de classe
}

Élise Palomares, Armelle Testenoire

\section{To cite this version:}

Élise Palomares, Armelle Testenoire. Indissociables et irréductibles : les rapports sociaux de genre, ethniques et de classe. L'Homme et la Société, 2010, 176-177, pp.15-26. 10.3917/lhs.176.0015 . halshs-01293209

\section{HAL Id: halshs-01293209 \\ https://shs.hal.science/halshs-01293209}

Submitted on 24 Mar 2016

HAL is a multi-disciplinary open access archive for the deposit and dissemination of scientific research documents, whether they are published or not. The documents may come from teaching and research institutions in France or abroad, or from public or private research centers.
L'archive ouverte pluridisciplinaire HAL, est destinée au dépôt et à la diffusion de documents scientifiques de niveau recherche, publiés ou non, émanant des établissements d'enseignement et de recherche français ou étrangers, des laboratoires publics ou privés. 


\section{INDISSOCIABLES ET IRRÉDUCTIBLES : LES RAPPORTS SOCIAUX DE GENRE, ETHNIQUES ET DE CLASSE}

Elise Palomares, Armelle Testenoire

\section{L'Harmattan | « L'Homme et la société »}

2010/2 n 176-177 | pages 15 à 26

ISSN 0018-4306

ISBN 9782296139381

Article disponible en ligne à l'adresse :

http://www.cairn.info/revue-l-homme-et-la-societe-2010-2-page-15.htm

\section{!Pour citer cet article :}

Elise Palomares, Armelle Testenoire, «Indissociables et irréductibles : les rapports sociaux de genre, ethniques et de classe », L'Homme et la société 2010/2 (n 176-177), p. 15-26. DOI 10.3917/lhs.176.0015

Distribution électronique Cairn.info pour L'Harmattan.

(C) L'Harmattan. Tous droits réservés pour tous pays.

La reproduction ou représentation de cet article, notamment par photocopie, n'est autorisée que dans les limites des conditions générales d'utilisation du site ou, le cas échéant, des conditions générales de la licence souscrite par votre établissement. Toute autre reproduction ou représentation, en tout ou partie, sous quelque forme et de quelque manière que ce soit, est interdite sauf accord préalable et écrit de l'éditeur, en dehors des cas prévus par la législation en vigueur en France. Il est précisé que son stockage dans une base de données est également interdit. 


\section{Indissociables et irréductibles : les rapports sociaux de genre, ethniques et de classe}

Une femme noire avec un enfant, laissée-pour-compte lors du passage du cyclone Katrina à la nouvelle Orléans, telle est l'image de la catastrophe qui apparaît sur les écrans de télévision ${ }^{1}$. Les deux côtés de l'Atlantique ont deux lectures de cette même réalité. En Amérique la plupart des commentateurs insistent sur la racialisation de la pauvreté, en France les observateurs du monde social sont en revanche rétifs à adopter la même grille de lecture. La pauvreté n'y est guère référée au racisme mais principalement aux rapports de classe. En tout état de cause, l'image n'apparaît ni aux yeux des uns ni à ceux des autres comme celle d'un être sexué. Or, la majorité des victimes de Katrina étaient des femmes, comme le sont également les salariées pauvres et les victimes de violences ${ }^{2}$. Cette femme abandonnée représente pour Judith Ezekiel une figure de l'intersectionnalité :

«C'est justement parce qu'elle se trouve au croisement des différents systèmes d'oppression qu'elle a été abandonnée à La Nouvelle-Orléans. ${ }^{3}$ »

Cet article fait écho à l'analyse fondatrice de l'intersectionnalité menée par Kimberlé W.Crenshaw ${ }^{4}$ qui met en évidence l'incapacité des politiques états-uniennes de lutte contre la violence envers les femmes à prendre en compte le caractère multidimensionnel de la subordination à laquelle les femmes de couleur sont soumises. Les dispositifs d'intervention,

1. Judith EZEKIEL, «Katrina à La Nouvelle-Orléans : réflexions sur le genre de la catastrophe », L'homme et la société, $\mathrm{n}^{\circ} 158,2005 / 4$, p. 189-199.

2. Jules FALQUET, De gré ou de force, les femmes dans la mondialisation, La Dispute, 2008,213 p.

3. Judith EZEKIEL, op. cit.

4. Kimberlé Williams CRENSHAw, « Mapping the Margins : Intersectionality, Identity Politics, and Violence Against Women of Color », in Martha AlberTson Fineman, Rixanne Myкiтıuk (eds.), The Public Nature of Private Violence, Routledge, New York, 1994, p. 93-118 ; Kimberlé Williams CRENSHAW, Demarginalising the Intersection of Race and Sex : A Black Feminist Critique of Antidiscrimination Doctrine, Feminist Theory and Antiracist Politics, The University of Chicago Legal Forum, Chicago, 1989, p. 154.

L’homme et la société, $\mathrm{n}^{\mathrm{o}}$ 176-177, avril-septembre 2010 
centrés sur une aide relationnelle et psychologique, ne prennent pas en compte les facteurs socioéconomiques ainsi que les discriminations en matière d'emploi et de logement ou encore la barrière de la langue qui pénalisent spécifiquement les femmes de couleur.

Ces analyses soulignent la nécessité d'adopter une grille de lecture multidimensionnelle qui prenne en compte les formes tant matérielles que symboliques des différents modes de subordination et la manière dont ceux-ci interagissent. La tendance est en effet forte, notamment en France, à imputer les inégalités matérielles au seul registre de la classe, considérant de ce fait implicitement que le sexisme ou le racisme n'auraient que des effets politiques et idéologiques. Or, l'apport des féministes matérialistes a montré que ces deux rapports de pouvoir - qui instituent les uns majoritaires, les autres minoritaires sur la base de marques physiques (sexe, couleur de peau...) ou culturelles (langue, religion...) - légitiment par ce système de référence symbolique un ordre hiérarchique qui se concrétise par un accès moindre aux ressources matérielles ainsi que par l'éviction des minoritaires des lieux de pouvoir et de la production des connaissances ${ }^{5}$.

\section{Un bien vieux renouvellement ${ }^{6}$}

Dans la perspective ouverte par ces travaux fondateurs, ce numéro entend contribuer à la réflexion engagée dans les sciences sociales francophones sur l'articulation entre les rapports de genre, ethniques et de classe. Irréductibles et indissociables, pour reprendre la formule d'Étienne Ba$\operatorname{libar}^{7}$, ils sont abordés comme des principes majeurs - transversaux de hiérarchie qui construisent l'ordre, le changement social et structurent les pratiques. Ce dossier reprend certaines communications proposées lors d'une journée d'études ${ }^{8}$ organisée conjointement par le RT $24^{9}$ de l'Association française de sociologie et le GRIS à l'université de Rouen en 2006, complétées par d'autres contributions de chercheuses françaises et canadiennes. Le dialogue qui se développe entre chercheur-e-s issu-e-s de la sociologie du genre et des rapports ethniques - ainsi qu'en témoigne ce

5. Colette Guillaumin, Sexe, Race et pratique du pouvoir ; l'idée de nature, CôtéFemmes, 1992, 239 p.; Nicole-Claude MATHIEU, L'anatomie politique ; catégorisations et idéologies du sexe, Côté-femmes, 1991,291 p.

6. Par analogie avec l'expression employée par Colette Guillaumin : «Un bien vieux néo-racisme », Vocabulaire historique et critique des relations interethniques, Pluriel Recherches, $\mathrm{n}^{\circ} 2,1994$, p. 1-3.

7. Étienne BALIBAR, « La construction du racisme », Actuel Marx, $\mathrm{n}^{\circ}$ 38, 2005.

8. Nous remercions Élise Lemercier pour sa contribution à l'organisation de ces journées d'études et Aude Rabaud pour la relecture de cet article.

9. Intitulé : «Genre, Classe, Race. Rapports sociaux et construction de l'altérité ». 
numéro - traduit le dynamisme de la réflexion actuelle. Ce dynamisme se manifeste également par les nombreux séminaires, colloques et publications organisés en France depuis les années 2000 par des centres et réseaux de recherche de ces deux champs de la sociologie : CEDREF ${ }^{10}$, URMIS, GTM, SIMONE-SAGESSE et le réseau « Race et Genre », RT 24 de l'AFS, EFIGIES, pour ne citer que les principaux.

Ce numéro s'inscrit dans la ligne éditoriale de L'homme et la société, initiée en 1991 par le dossier intitulé - avec un brin d'ironie - «Femmes et sociétés », qui analysait la situation et les engagements des femmes en matière de sexualité, de mariage et d'avortement en lien avec les antagonismes de classe dans des sociétés «marquées majoritairement par le nationalisme, qu'il soit allemand, algérien, français, iranien ou encore bangladeshi ${ }^{11} \gg$. Dans ce numéro de 1991, Liliane Crips cite les propos d'un représentant de la droite nationaliste allemande de l'entre-deux-guerres qui déplore que :

«La "femme émancipée" revendique le droit d'avoir des relations avec des nègres, des juifs, des chinois ; au lieu d'être par vocation la gardienne de la race, la femme se transformerait ainsi, par l'émancipation, en destructrice des fondements mêmes de la race. ${ }^{12}$ »

Antiféminisme et racisme doctrinaires, énoncés dans le même souffle, rappellent, par la caricature, combien la réflexion sur l'intersectionnalité est incontournable tant pour saisir la fabrique de l'ordre social en ellemême que les idéologies visant à le perpétuer ou à le changer. Quinze ans plus tard, avec le dossier "Féminisme, conflits, mouvements », Marc Bessin et Elsa Dorlin soulignent l'engagement de la revue « vis-à-vis des savoirs et des pratiques développés depuis sur les questionnements féministes, de genre et de sexualité, pour en être à la fois le relais, la caisse de résonance, et pourquoi pas l'avant-poste ${ }^{13} \gg$. La troisième vague du féminisme se saisit aujourd'hui de questionnements qui la situent dans un dialogue critique avec des problématiques anglo-saxonnes.

10. Jules FALQUET, Emmanuelle Lada et Aude Rabaud proposent une recension précise et détaillée des différents lieux, moments, productions et protagonistes de ces développements, cf. Cahiers du Cedref, "(Ré)articulation des rapports sociaux de sexe, classe et "race" : repères historiques et contemporains ", 2006, p. 7-29.

11. Monique SELIM, «Enjeu ou otage : la "femme" aux prises avec la société »; Introduction au dossier «Femmes et sociétés », L’homme et la société, n ${ }^{\circ} 99-100,1991$, p. 3-23.

12. Alfred Rosenberg, Der Mythus des 20. Jahrhunderts, Münich, 1934, p. 514.

13. Elsa DoRLin et Marc BESSIN, «Les renouvellements générationnels du féminisme : mais pour quel sujet politique ?», L'homme et la société, $\mathrm{n}^{\circ}$ 158, 2005, p. 11-25. 
Silence... Les minoritaires peuvent-elles parler?

Dès les années 1970, le black feminism aux États-Unis, puis les postcolonial studies et subaltern studies aux Suds poseront les jalons d'une analyse appréhendant la hiérarchie comme un système complexe où se conjuguent les rapports ethniques, de genre et de classe ${ }^{14}$. Le black feminism oppose à la conception moniste du féminisme hégémonique [des femmes blanches des classes moyennes] une théorie du «point de vue situé » qui s'ancre dans l'expérience spécifique des minoritaires ${ }^{15}$. Ainsi que l'affirme le Combahee River Collective ${ }^{16}$ :

\footnotetext{
«Nous avons du mal à séparer les oppressions de race, de classe et de sexe, parce que fréquemment dans nos vies, nous en faisons l'expérience simultanée. ${ }^{17}$ »
}

La production de connaissance s'inscrit dans ces rapports. De ce fait, toute connaissance est située, en ce sens qu'elle est liée aux positions et aux manières d'interpréter le monde de ceux qui la produisent et la valident, même si elle ne se présente pas toujours comme telle. Le travail réflexif du chercheur-e sur sa propre position et le caractère collectif du savoir produit permettent de «faire bouger le langage de l'institution universitaire ${ }^{18} »$. Danièle Kergoat rappelle dans l'entretien qui ouvre ce dossier que, si son questionnement de départ est issu de l'observation de longue durée qu'elle a menée dans les entreprises, ses analyses témoignent de son engagement dans trois mouvements sociaux en France : le soutien au peuple algérien, le syndicalisme et le mouvement féministe. Qu'il s'agisse du travail avec les ouvrières de Lip ou de formations syndicales, elle soumet ses analyses à la discussion des salariées. Cet engagement pluriel lui permettra de sortir du «tout est explicable par le rapport de classe », alors en vigueur, pour penser dès les années $1970{ }^{19}$ l'imbrication des rapports sociaux.

14. Elsa DORLIN, « De l'usage épistémologique et politique des catégories de "sexe" et de "race" dans les études sur le genre », Cahiers du genre, n 9, 2005, p. 83-105.

15. Christian POIRET, «Articuler les rapports de sexe et interethniques : quelques enseignements du débat nord-américain », Revue Européenne des Migrations Internationales, 2005 (21)1, p. 195-226.

16. Groupe féministe et socialiste Noir de Boston qui se réunira de 1974 à 1980. Pour un historique cf. Jules FALQUET, « Le Combahee River Collective, pionnier du féminisme noir ; contextualisation d'une pensée radicale », Cahiers du Cedref, 2006, p. 69-104.

17. Combahee River Collective, "Déclaration du Combahee River Collective », Cahiers du Cedref , 2006, p. 53-67.

18. Patricia Hill Collins, «La construction sociale de la pensée féministe Noire », in Elsa DoRLIn, Black Feminism ; anthologie du féminisme africain-américain, 1975-2000, L'Harmattan, Paris, 2008, p. 173.

19. Danièle KergOAT, Les ouvrières, Le Sycomore, Paris, 1982, 141 p. 
Aux États-Unis, le black feminism fondera une épistémologie alternative qui place l' expérience des femmes noires américaines au cœur de la production de connaissances et la politise ${ }^{20}$. Le black feminism et les $s u$ baltern studies poseront ainsi la question de la (dé)colonisation des catégories de la connaissance et, partant, celle de la capacité d'expression des minoritaires ${ }^{21}$. Audre Lorde revendique une parole émancipatrice animée par « la volonté de nous définir, de nous nommer, de parler en notre nom, et pas que les autres nous définissent et parlent à notre place ${ }^{22} »$. Les raisons du silence des minoritaires sont puissantes ; elles sont liées à la peur de s'exposer au mépris et au jugement des majoritaires. La prégnance de la catégorisation enferme les minoritaires dans un groupe clos sur luimême et ce en dépit de la diversité des situations et des parcours qui sont les leurs ${ }^{23}$. Comment, en effet, parler du caractère arrangé de son mariage avec une enquêtrice occidentale sans courir le risque d'être perçue comme une victime soumise à l'ordre patriarcal ? Comment être porte-parole d'un syndicat sans se heurter à des réactions racistes et sexistes des propres membres de son syndicat? Dans sa contribution au présent numéro, Corinne Mélis montre néanmoins comment, malgré les obstacles, les minoritaires peuvent parler même si leur légitimité à représenter l'universel n'est jamais définitivement acquise. Une de ses enquêtées se remémore sa première prise de parole en tant que déléguée syndicale :

«Dans la salle il n'y avait pas un black, pas un beur. On rigolait avec le collègue qui me soutenait et qui est homo, la beur et l'homo main dans la main. Et on aurait dû dire que j'étais lesbienne, comme ça c'était la totale : beur, femme, syndicaliste et lesbienne !»

Les arènes de délibération sont traversées par les processus de domination, marginalisant l'expérience et la parole des minoritaires. Discutant le concept d'espace public de Jürgen Habermas, Nancy Fraser défend l'existence d'espaces de délibération alternatifs, qu'elle qualifie de contrepublics subalternes, où les minoritaires élaborent une connaissance et des

20. Patricia Hill COllins, op. cit.

21. Chandra Mohanty, «Under Western Eyes : Feminist Scholarship and Colonial Discourses », Feminist Review, n³0, 1988, p. 61-88 ; Gayatri CHAKRAVORTY SPIVAK, «Can the Subaltern Speak ? », in Cary NELSON and Lawrence GrossberG (ed.), Marxism and the Interpretation of Culture, Chicago, University of Illinois Press, 1988, p. 271-313, (Les Subalternes peuvent-elles parler?, traduction française de Jérôme Vidal, Éditions Amsterdam, 2006).

22. Audre LORDE, «Transformer le silence en paroles et en actes », in Elsa DoRLIN, Black Feminism ; anthologie du féminisme africain-américain, 1975-2000, op . cit., p. 7480 .

23. Colette Guillaumin, L’idéologie raciste, Gallimard, Folio, Paris, 2002, 378 p. 
revendications propres. Ces contre-publics se constituent en réaction aux processus de marginalisation qui sont à l'œuvre dans l'espace public. Ils n'ont cependant pas pour objectif, à terme, le séparatisme ; celui-ci n'est qu'une étape préalable à l'action publique. Ils ont deux fonctions qui sont inextricablement liées :

«D'une part, ils fonctionnent comme des espaces de repli et de regroupement ; d'autre part, ils fonctionnent comme des bases et des terrains d'essai pour des activités dirigées vers des publics plus larges. C'est précisément dans la dialectique entre ces deux fonctions que réside leur potentiel émancipateur. ${ }^{24}$ »

Le syndicalisme et le féminisme ont cette ambition. Ils peinent néanmoins à prendre en compte l'ensemble des rapports de domination. Danièle Kergoat relate la cécité du syndicalisme des années 1970 aux rapports ethniques et de sexe et à la manière dont ceux-ci configurent le rapport de classe. Dans les entreprises qu'elle observe, les clivages internes à la classe ouvrière apparaissent décisifs pour comprendre les pratiques ouvrières et la dynamique des conflits. Les rapports de sexe ne viennent pas colorer $a$ posteriori le rapport de classe, ils participent à sa construction, produisant « une manière homme ou une manière femme d'être ouvrier ${ }^{25}$ ». C'est dans la simultanéité et non dans l'addition que se conjuguent les rapports sociaux. Se pose dès lors la construction d'un paradigme véritablement multidimensionnel qui permette de penser l'hétérogénéité des classes sociales, du groupe des femmes mais aussi de celui des groupes racisés sans les réifier en de multiples sous-catégories ${ }^{26}$.

\section{L'intersectionnalité}

L'intersectionnalité porte cette ambition paradigmatique, et ce en dépit des critiques épistémologiques ${ }^{27}$ et des méfiances politiques croisées dont elle fait l'objet, affirme Sirma Bilge ${ }^{28}$. Outre une revue critique et docu-

24. Nancy FRASER, Qu'est-ce que la justice sociale? Reconnaissance et redistribution, La Découverte, Paris, 2005, p. 128.

25. Danièle KERGOAT, Les ouvrières, op. cit.

26. Elsa DORLIN, «Les renouvellements générationnels du féminisme : mais pour quel sujet politique? », op. cit.

27. Ibidem; Elsa DoRlin (éd.), Sexe, race, classe. Pour une épistémologie de la domination, PUF, Paris, 2009, 313 p.; Danièle KERGOAT, «Dynamique et consubstantialité des rapports sociaux », in Elsa DoRlin (éd.), Sexe, race, classe. Pour une épistémologie de la domination, ibidem, p. 111-125.

28. Nous tenons à remercier chaleureusement Sirma Bilge pour l'emprunt que nous avons fait à sa contribution pour l'intitulé du présent dossier. Professeur adjoint à l'université de Montreal, Sirma Bilge dirige le pôle de recherche Intersectionnalité du Centre d'études ethniques des universités montréalaises (CEETUM) qui se propose de constituer 
mentée de ces multiples mésusages, 1'article de Sirma Bilge explore le potentiel de transformation de la pensée sociologique qu'ouvre l'intersectionnalité, se proposant de la «situer par rapport aux autres modes de penser l'ordre social, ses divisions et hiérarchies » et leurs transformations. Au-delà de son usage fédératif dans les recherches anglo-saxonnes - un mot-clé qui permet d'identifier revues, centres de recherche et colloques - la pensée intersectionnelle développe une critique du raisonnement analogique et dépasse le monisme - qu'il soit marxiste ou féministe - qui donne le primat à un rapport social donné. L'intersectionnalité prend le relais du pluralisme cumulatif, qui, de l'addition à la multiplication, a produit des modèles mathématiques sophistiqués ${ }^{29}$ fondés sur une prémisse erronée, selon laquelle les rapports sociaux seraient indépendants les uns vis-à-vis des autres. Soulignant la consistance de cette démarche holiste, Sirma Bilge pointe cependant les dangers d'un durcissement idéologique et discerne des directions divergentes de la recherche selon le degré d'autonomie relative accordé à chacun des rapports de pouvoir. Cette définition de l'intersectionnalité n'est néanmoins pas la seule approche à être mobilisée dans les analyses contemporaines. L'article de Danielle Juteau montre, en effet, que l'intersectionnalité est plus fréquemment celle des identités que celle des rapports sociaux, masquant ainsi la domination masculine que celle-ci soit le fait des majoritaires ou des minoritaires. L'enjeu décisif est, pour Danielle Juteau, de comprendre la constitution des catégories sociales que sont la classe, le sexe, l'ethnie ou la race par les rapports de pouvoir. «Le genre précède le sexe ${ }^{30}$ », au sens où les rapports de pouvoir qui sont causalement premiers s'appuient sur des marques telles que le sexe biologique, la couleur de la peau ou la culture. L'articulation ne peut être pensée qu'en remontant théoriquement aux trois rapports de pouvoir qui instituent les un-e-s majoritaires, les autres minoritaires. Chacun de ces rapports de pouvoir a des conséquences matérielles et sa propre historicité. L'hétérogénéité du groupe des femmes est alors conciliable avec une théorisation de leur commune oppression ; celle-ci revêtant des formes concrètes plurielles qui génèrent des expériences également diversifiées (et parfois contradictoires). Le sujet collectif «fem-

une plateforme de production et d'échanges des savoirs sur les articulations de l'ethnicité, comme vecteur des relations et des divisions sociales, avec d'autres catégories et rapports structurants l'ordre social tels la classe, le genre, la race et l'âge.

29. West CANDACE and Sarah FenstermaKeR, «Doing Difference », Gender and Society, 9 (1), 1995, p. 8-37.

30. Christine DelPhy, L'ennemi principal, tome 2 : Penser le Genre, Syllepse, Paris, 2001, p. 251. 
mes », tel que le théorise Colette Guillaumin ${ }^{31}$, n'est pas un donné de l'expérience mais un objectif d'ordre politique. Par analogie avec Kimberlé W. Crenshaw qui conceptualise la race comme une coalition entre hommes et femmes de couleur, Danielle Juteau propose de penser le sexe « comme une coalition entre femmes de race, de classe et d'orientation sexuelle différentes qui, au-delà de leurs expériences concrètes différentes, ont en commun leur appropriation individuelle et collective ». Un tel sujet collectif n'est néanmoins pas donné a priori, ainsi que le rappelle Danièle Kergoat. Il se construit dans et par le conflit lui-même avec tous les freins générés par les rapports de pouvoir.

Cette définition de l'intersectionnalité pose le cadre paradigmatique dans lequel s'inscrivent les contributions de ce numéro. Dès lors, se pose la question récurrente des méthodes susceptibles d'appréhender les effets croisés des systèmes de pouvoir. Danièle Kergoat met en garde contre toute analyse «en surplomb»; elle invite à partir des pratiques dans toute leur complexité, en veillant au respect des principes matérialiste et d'historicité appliqués à chacun des rapports sociaux. La méthode biographique, mobilisée par Blandine Veith, vise à appréhender de manière dynamique l'entrecroisement des rapports sociaux et leurs effets sur les parcours individuels. Les récits biographiques renvoient à plusieurs sphères de l'existence qui s'entrecroisent dans les parcours de femmes migrantes engagées dans des associations féminines et multiculturelles. Blandine Veith s'interroge sur les silences qui émaillent le cours des récits. Elle montre qu'ils sont partie prenante de la narration et, de ce fait, à intégrer à part entière dans l'analyse. Loin d'être liés à des défaillances de la mémoire, ils correspondent à des épisodes cruciaux des parcours de vie où s'exercent de manière aiguë les rapports de domination. Le croisement des récits de vie entre eux et avec des données d'observation apparaît dès lors décisif pour analyser l'étroite intrication des rapports ethniques, de classe, de genre mais également de génération qui se co-construisent mutuellement. La saisie méthodologique de l'imbrication des rapports sociaux est également au cœur de la contribution d'Alain Frickey et Jean-Luc Primon. À partir des données de l'enquête «Génération 98 » du Cereq, ils analysent les parcours scolaire et professionnel des jeunes hommes et femmes issus de l'immigration nord-africaine. Par opposition aux traitements statistiques « toutes choses égales par ailleurs » qui neutralisent certaines variables pour isoler les effets «en soi » de l'une d'entre elles, ils montrent que les jeunes sont placés simultanément dans trois rapports sociaux qui ne peuvent être dissociés. Les parcours des jeunes sont profondément

31. Colette Guillaumin, Sexe, Race et pratique du pouvoir, op. cit. 
marqués par la trajectoire migratoire de leurs parents et leur origine ouvrière, liées à l'histoire ouvrière et coloniale, et ce y compris pour les diplômés du supérieur. Cette position minoritaire se configure néanmoins de manière différenciée selon le sexe.

\section{Actualité du matérialisme}

Le mouvement féministe a sans conteste été à l'origine de la pensée intersectionnelle, en raison des tensions récurrentes entre la lutte antisexiste et antiraciste, que ce soit aux États-Unis ${ }^{32}$ ou plus récemment en France ${ }^{33}$. Jules Falquet rappelle l'apport conceptuel du féminisme matérialiste, ses sources et son postulat central : la différence entre homme et femme n'existe pas en soi, elle dérive d'un rapport social (fait d'appropriation physique directe), rapport de pouvoir spécifique que les lesbiennes remettent précisément en cause dès lors qu'elles débordent les limites de « pratiques sexuelles strictement privées et invisibles ${ }^{34}$ ». L'approche matérialiste implique de ne pas reléguer le genre et la race au registre idéologique et suppose une volonté de penser ensemble les inégalités complexes et les différences qu'elles produisent ${ }^{35}$.

Comme le rappelle ici Danielle Juteau, la difficulté à penser l'articulation des rapports sociaux dans le cas français est notamment liée à l'impensé de la domination ethnique ${ }^{36}$ issue de l'histoire spécifique de la France. Certes, les travaux pionniers de Roger Bastide ${ }^{37}$ sur les « Amériques noires » ouvriront la voie dès les années 1940 à une triple réflexion sur les processus d'adaptation à des contextes sociohistoriques multiples, l'articulation entre faits culturels et sociaux et les relations de pouvoir; ses travaux n'auront néanmoins qu'un impact limité en France. Ce n'est que

32. Christian POIRET, 2005, op. cit.

33. Nacira GUENIF-SOUILAMAS et Éric MACE, Les féministes et le garçon arabe, Éditions de l'Aube, 2004, 107 p.; Christelle HAMEL, «La sexualité entre sexisme et racisme : descendant-e-s de migrant-e-s originaires du Maghreb et la virginité », Nouvelles Questions Féministes, vol. 25, $\mathrm{n}^{\circ} 1,2006$; Christine DELPHY, « Antisexisme ou anti-racisme? Un faux dilemme », Nouvelles Questions Féministes, vol. 25, n 1, 2006.

34. Jules FALQUET, « Rompre le tabou de l'hétérosexualité, en finir avec la différence des sexes : les apports du lesbianisme comme mouvement social et théorie politique », Genre, sexualité et société, $\mathrm{n}^{\circ} 1,2009$.

35. Véronique De RudDER, Christian POIRET et François VOuR'CH, L'inégalité raciste, l'universalité républicaine à l'épreuve, Paris PUF, 2000, 211 p.

36. Pour un état des lieux récent cf. Nicolas Jounin, Elise PALOMAREs et Aude RABAUD, «Introduction au dossier "Ethnicisations ordinaires, voix minoritaires" », Sociétés contemporaines, $\mathrm{n}^{\circ} 70,2008$, p. 7-23.

37. Denys CuCHE, «L'étude des relations interethniques dans l'œuvre de Roger Bastide », Bastidiana, juillet-décembre 1998, n² 23-24, p. 137-166. 
depuis les années 1970 que se développe une littérature francophone, aujourd'hui abondante. Le fait ethnique a en effet longtemps été conçu en France comme une importation a-critique, voire frauduleuse, d'une pensée, de catégories et d'une histoire états-unienne, pays dans lequel, à l'inverse du cas français, «la naissance de ce domaine d'étude est contemporaine de l'institutionnalisation de la sociologie comme discipline universitai$\mathrm{re}^{38} »$. Pour Denys Cuche, les causes du retard français sont à la fois idéologiques et épistémologiques: le silence de Durkheim quant aux faits ethniques a joué un rôle-clé, relayé par la suite par la prédominance du marxisme puis du structuralisme. Denys Cuche relève par exemple que, parti aux États-Unis découvrir l'école de Chicago, «Halbwachs, qui rédige son article [opposant Chicago à Paris] dans une période où la France connaît le plus fort taux d'immigration du monde, n'imaginait pas que $\mathrm{Pa}$ ris, ou une autre métropole française, Marseille par exemple, puisse également offrir un laboratoire approprié pour examiner une "expérience ethnique" ». Longtemps négligée par la recherche, la place du religieux dans la production de catégories de l'altérité et leur contestation ${ }^{39}$ fera l'objet d'un renouvellement des perspectives à partir de 1995, interrogeant notamment la place de l'islam dans les réarrangements entre les sexes en situation minoritaire ${ }^{40}$.

Par un curieux retournement, la classe qui fut naguère l'explication «en dernière instance » finit parfois par être négligée dans les travaux contemporains. Certes, l'absence de prise en compte de ce qu'Emmanuelle Retaillaud-Bajac ${ }^{41}$ appelle " "les enjeux de classe”, c'est-à-dire sociaux, professionnels et financiers » reste exceptionnelle. Néanmoins, le sens parfois très général donné au mot "classe » rabat celle-ci sur les inégalités sociales sans nécessairement la saisir comme un rapport social s'enracinant dans la production. L'analyse menée par Corinne Mélis est pertinente à cet égard, et ce à plus d'un titre. Par son objet : rares sont les travaux portant sur le syndicalisme des femmes migrantes et des descendants d'im-

38. Denys Cuche, «Roger Bastide, le "fait individuel" et l'école de Chicago », $\mathrm{Ca}$ hiers internationaux de sociologie, vol. 1, $\mathrm{n}^{\circ}$ 124, 2008, p. 41-59.

39. Nathalie KAKPO, L'islam, un recours pour les jeunes, Presses de Science Po, 2007 ; Françoise LORCERIE (éd.), La Politisation du voile. L'affaire en France et son écho à l'étranger, Paris, L'Harmattan, coll. « Confluences Méditerranée », 2005, p. 37-52.

40. Simona TERSIGNI, «Jalons pour une lecture imbriquée du genre et du religieux dans le champ des migrations et des relations interethniques en France », Cahiers $d u C e$ dref, 2008, p. 251-273.

41. Emmanuelle Retaillaud-BAJAC, «Du “clan divin des femmes amoureuses" à la "race maudite" : élaboration, représentations et discontinuités de l'identité lesbienne dans la trajectoire de Mireille Havet (1898-1932) », Genre, Sexualité et société, n 1, printemps 2009. 
migrés, sans doute parce que ce thème déborde l'imagination sociologique ordinaire, elle-même nécessairement tributaire des idées dominantes de l'époque sur ces femmes - entre la figure de la victime et l'égérie de l'intégration. Par son analyse, à partir de l'exposé des conditions de leur engagement liées à leur place dans la division sexuelle et ethnique du travail, Corinne Mélis décrit finement la complexité des motifs de leur engagement, en particulier le rôle singulier du sexisme et du racisme au et hors travail. Elle revisite le problème récurrent de l'hétérogénéité de la classe ouvrière à partir de la situation de ces femmes. Pour elle, la diversité des positions et des situations au sein du salariat et les éventuels conflits d'intérêts entre «fractions de classe » n'impliquent pas pour autant la fin des rapports et conflits de classe. Ces distinctions analytiques sont déterminantes pour la connaissance comme pour l'action : les difficultés d'une commission «femmes » au sein d'un syndicat clivant les «cheminotes » d'un côté et les «femmes du nettoyage» de l'autre soulignent « les difficultés d'agir si la coextensivité des pouvoirs n'est pas considérée $»$. Clivages et concurrences entre catégories de main-d'œuvre prennent des formes contrastées selon les secteurs, l'état local du marché du travail et les formes de discrimination racistes et sexistes pratiquées par les employeurs comme mode de gestion de la main-d'œuvre.

Données quantitatives à l'appui, Marguerite Cognet étudie la dimension internationale de la division genrée et ethnoracialisée des soins et des services à la personne. Elle interroge en particulier le rôle des États français et canadien dans ce processus, politiques sanitaires et politiques migratoires se combinant pour « confiner les femmes noires dans les emplois de la domesticité et des services ». Au tournant des années 1980, avec l'externalisation, Marguerite Cognet décrit l'émergence d'un «marché secondaire, comme un segment du marché du travail caractérisé par la déqualification, l'insécurité d'emploi, les mauvaises conditions d'exercice, un turn-over élevé, des règles de fonctionnement et d'avancement arbitraires et l'absence de soutien syndical ». Par exemple, en France, les emplois créés en 1989 pour la garde d'une famille ou d'un patient $(24 \mathrm{~h} / 24$, semaine et jours fériés inclus) sont en totalité occupés par des femmes, et parmi elles, $46 \%$ sont nées dans un pays d'Afrique ou dans les DOM. Au Québec, plusieurs programmes se sont succédé : dans les diverses conditions drastiques du dernier, l'aide familiale n'a pas le droit d'exercer un autre emploi et ne peut travailler que pour l'employeur qui est nommé sur son permis de travail.

Dans le cas des femmes de chambre étudié par Armelle Testenoire, dans le Nord et en Normandie, sur un marché du travail tendu, les autoch- 
tones de milieu populaire sont recrutées en priorité sur les femmes allogènes (ou supposées telles en fonction de la couleur de la peau). Dans l'ordinaire des relations de travail, l'auteure montre comment frontière sociale et frontière ethnique se superposent et se renforcent mutuellement : alors que les employés en contact direct avec la clientèle parviennent à former un collectif de travail, les femmes de chambre, chargées du « sale boulot » en étage, sont isolées, invisibilisées et niées. Elles n'ont pas de perspective de carrière : "close sur elle même », la catégorie "migrante » cadenasse leur avenir professionnel. Cette contribution vient étayer la critique d'une conception étroitement intersubjective de la reconnaissance en montrant comment les employeurs organisent l'invisibilité des femmes de chambre, visant à les faire "disparaître en tant qu'individues pour ne devenir qu'une fonction ». Armelle Testenoire analyse ainsi comment l'injustice sociale se déploie dans toute sa complexité économique, symbolique et culturelle à travers des lieux, des modes de gestion, des procès de travail, des catégories et des échanges quotidiens entre employeurs, salariés et clients. Articulant les paradigmes de la reconnaissance et de la redistribution, elle propose une conception dialectique de l'injustice sociale où le déni de reconnaissance s'articule étroitement à une subordination économique, qui en retour l'étaye.

En conclusion, nous souhaiterions revenir sur l'usage du terme intersectionnalité. Dans cette introduction, nous avons veillé à mettre ce terme en italique en raison du débat sur son usage au sein des sciences sociales en France. Le travail engagé autour de ce numéro nous a permis d'en clarifier les enjeux. Au terme de ce travail, nous voudrions souligner le potentiel de transformation de la pensée sociologique qu'il représente en tant que cadre paradigmatique pour appréhender la différenciation et les inégalités sociales dans toute leur complexité. Certes l'intersectionnalité recouvre des usages pluriels, elle possède néanmoins une pertinence heuristique sous réserve qu'elle réfère aux rapports sociaux de pouvoir appréhendés dans une perspective dynamique, au sens que lui donne Georges Balandier. Puisse ce numéro contribuer au débat sociologique engagé sur cette question... Laissons maintenant la parole aux auteur-e-s.

Elise PALOMARES et Armelle TESTENOIRE

* * *

